\title{
Immune checkpoint inhibitor-induced myocarditis in lung cancer patients: a case report of sintilimab-induced myocarditis and a review of the literature
}

\author{
Huanhuan Bi, Dunqiang Ren, Qiang Wang, Xiaoqian Ding, Hongmei Wang \\ Department of Respiratory and Critical Care Medicine, The Affiliated Hospital of Qingdao University, Qingdao, China \\ Correspondence to: Hongmei Wang. Department of Respiratory and Critical Care Medicine, The Affiliated Hospital of Qingdao University, Qingdao \\ 266000, China. Email: dor.whm@163.com.
}

\begin{abstract}
Since its initial approval by the United States Food and Drug Administration (FDA) in 2014, the indications for the use of the immune checkpoint inhibitors (ICIs) in non-small cell lung cancer (NSCLC) patients has increased. However, to date, there has no report on immune myocarditis caused by the ICI sintilimab. In addition, there has been no literature review on ICI-induced myocarditis in lung cancer patients. This is a case report of an elderly male patient who presented with a productive cough and progressive dysphagia for 3 days. The symptoms started on day 6 after the third cycle of sintilimab treatment for his lung carcinoma. In accordance with his clinical manifestations of progressive dysphagia, a previous history of lung cancer, abnormal electrocardiograph, significantly increased serum myocardial enzyme levels, and normal coronary angiography results, sintilimab-induced myocarditis was diagnosed. Methylprednisolone (80-40 mg) was used to inhibit the immune injury and the patient was safely discharged on the 13th day following admission. Since ICI-induced myocarditis is rare and fatal, we summarized the characteristics of 20 cases of the disease in lung cancer patients to highlight to oncologists, respiratory experts, and cardiologists the serious side effects of the drug when they encounter lung cancer patients using ICIs. Like most ICIs, sintilimab induces severe immune myocarditis and requires corticosteroids therapy, and this should be recognized by doctors in multiple departments.
\end{abstract}

Koywords! Lung cancer; myocarditis; immune checkpoint inhibitor (ICI); corticosteroids; case report

Submitted Oct 30, 2020. Accepted for publication Jan 17, 2021.

doi: 10.21037/apm-20-2449

View this article at: http://dx.doi.org/10.21037/apm-20-2449

\section{Introduction}

Sintilimab is an immune checkpoint inhibitor (ICI), known to activate cytotoxic $\mathrm{T}$ lymphocytes and improve their antitumor activity by inhibiting the binding of programmed cell death 1 (PD-1) ligands on tumor cells [programmed cell death ligand 1 (PD-L1) and PD-L2] to the PD-1 receptors on T cells (1). Increased used of ICIs in a variety of malignancies has led to a subsequent increase in the proportion of patients eligible for ICIs, from $1.5 \%$ in 2011 to $43.6 \%$ in 2018 (2). Despite the benefits, immune-related adverse events (irAEs) have been detected more frequently than initially estimated (3). These include, but are not limited to, endocrinopathies, hepatitis, pneumonitis rash, and colitis (4). Amongst these, ICI-induced myocarditis is of particular concern. Despite a reported incidence of less than $1 \%$, it is considered one of the most serious complications due to a high mortality rate of up to $50 \%(5-7)$. The present study highlights the clinical importance of early recognition of this relatively uncommon but fatal complication of sintilimab.

In this investigation, the clinical characteristics, diagnosis, and treatment of 20 cases of ICI-induced myocarditis in patients with lung cancer were reviewed and summarized in Table 1 (8-25). We present the following article in accordance with the CARE reporting checklist (available at http://dx.doi.org/10.21037/apm-20-2449). 


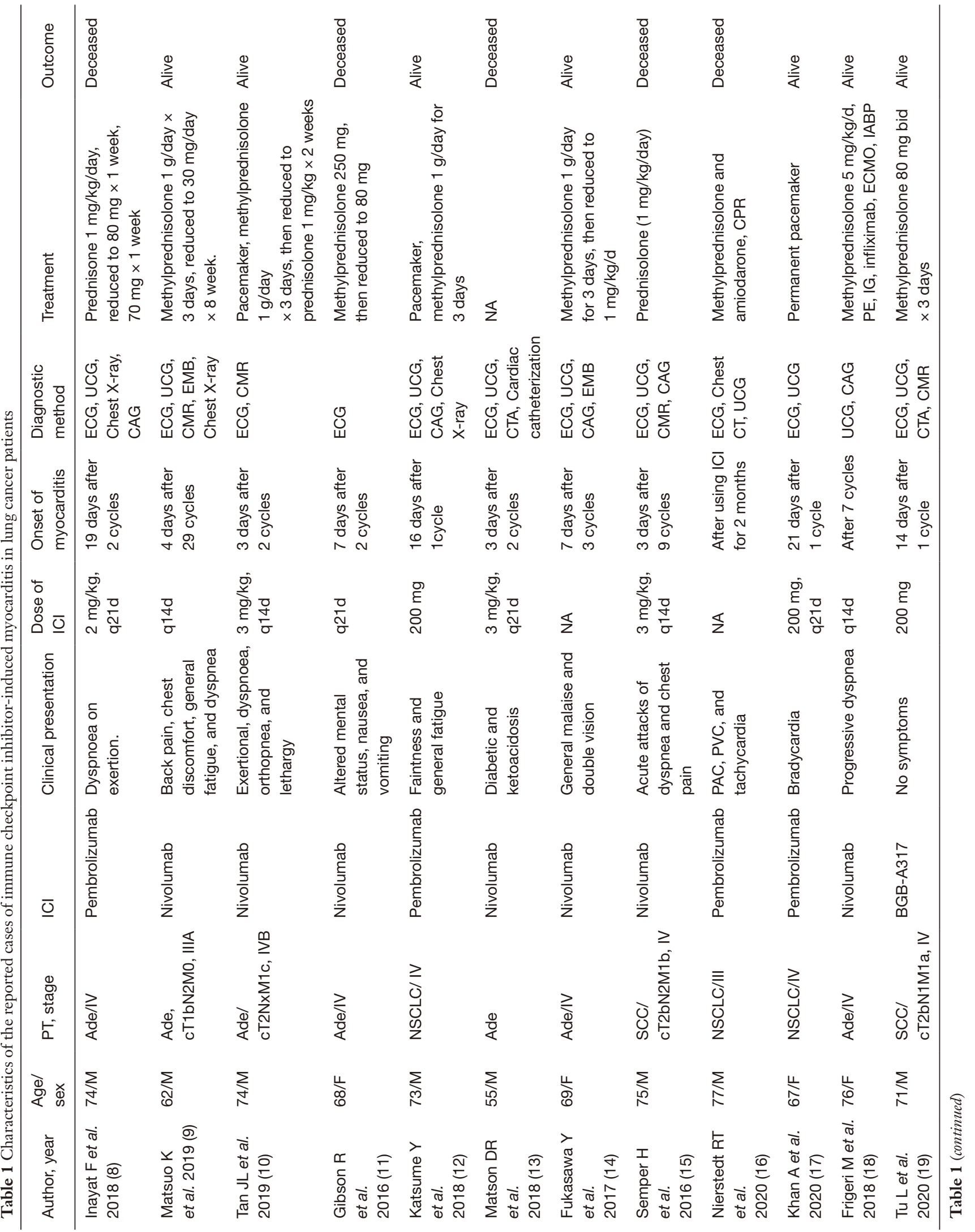




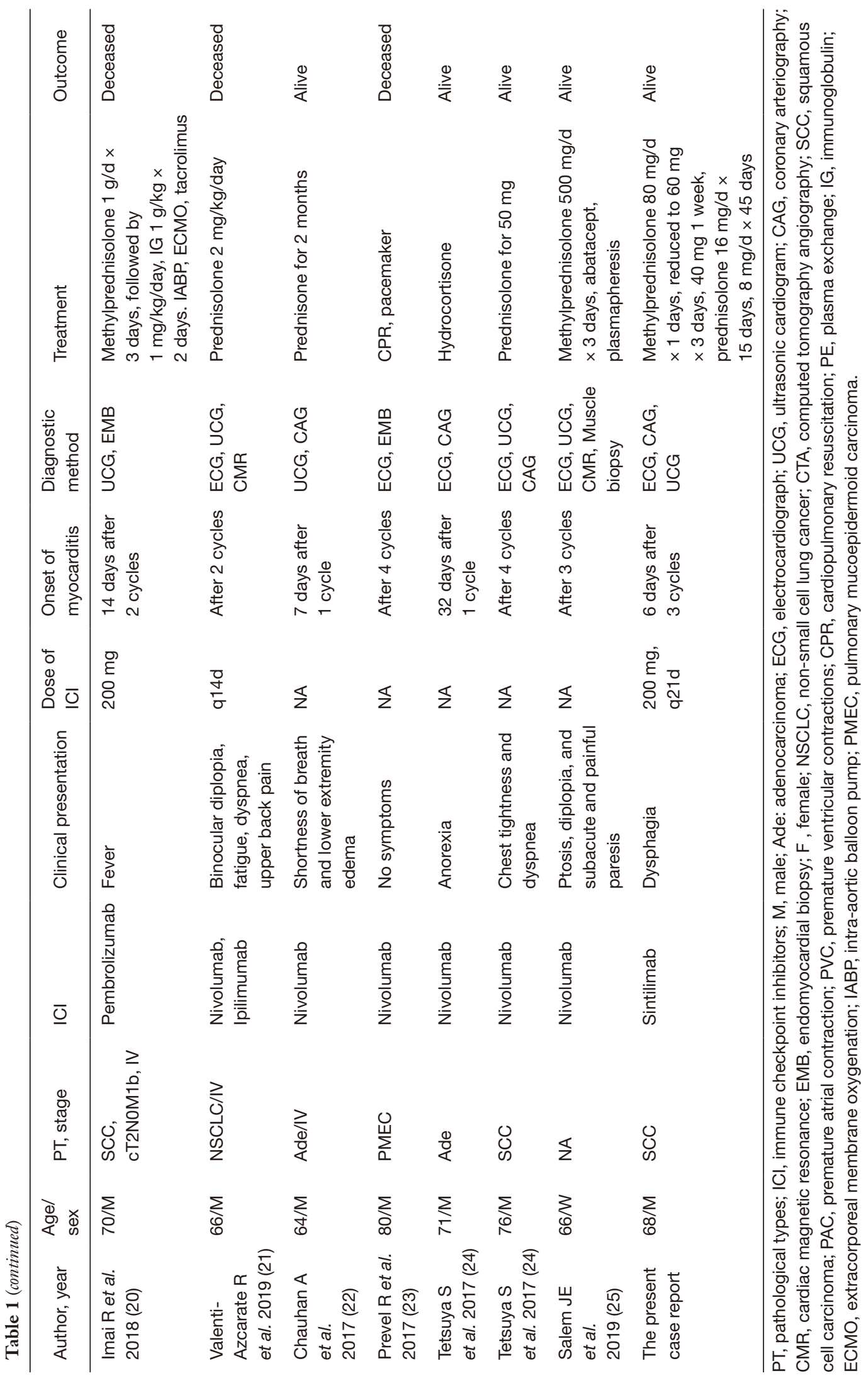




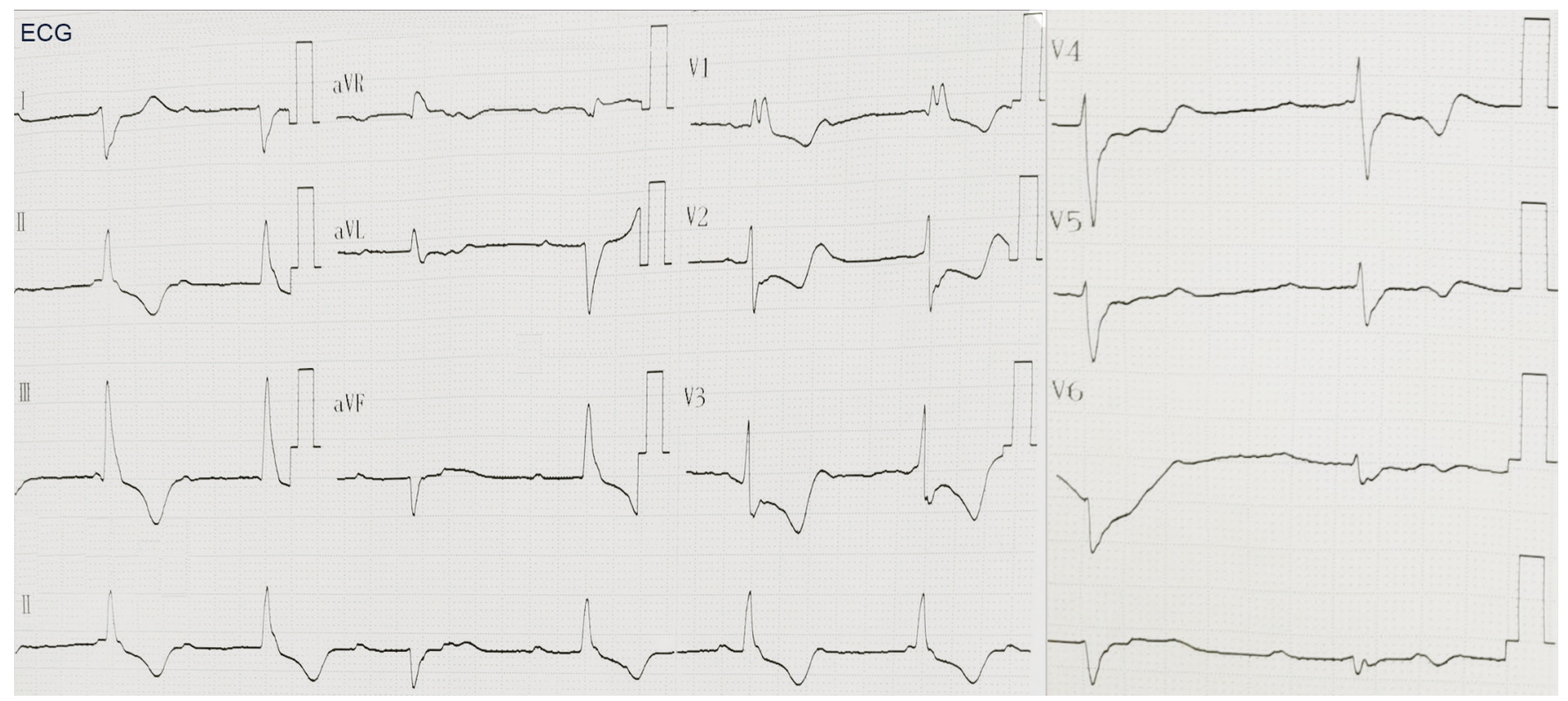

Figure 1 Electrocardiogram showing idioventricular rhythm, complete right bundle branch block, second-degree atrioventricular block, and diffuse ST segment depression.

\section{Case presentation}

A 68-year-old Asian male was admitted to the Affiliated Hospital of Qingdao University presenting with a history of productive cough and progressive dysphagia for the past three days. These symptoms appeared on day 6 after the third cycle of intravenous sintilimab (200 mg, q21d). The patient did not report chest tightness, chest pain, dyspnea, wheezing, hemoptysis, syncope, or peripheral edema. Thirty years ago, he had a history of smoking approximately 24 packets of cigarette per year for 10 years.

Two months ago, the patient was diagnosed with nonsmall cell lung cancer (NSCLC). It was classified as a poorly differentiated squamous cell carcinoma, cT3N3M0, stage III $\mathrm{b}$, and the programmed cell death ligand 1 (PD-L1) tumor proportion score (TPS) was 60\% (as assessed by the Dako PD-L1 IHC 22C3 pharmDx kit). The patient initially received two cycles of chemotherapy with carboplatin and paclitaxel. As recent studies have suggested that the combination of immunotherapy with chemotherapy could improve the survival rate of patients with lung cancer (26), the patient was also administered immunotherapy with sintilimab.

At admission, the patient's vital signs were unstable, with a temperature of $36.3{ }^{\circ} \mathrm{C}$, irregular heart rate of $40-50$ beats per minute, a respiratory rate of 17 breaths per minute, low blood pressure of $109 / 70 \mathrm{mmHg}$, and oxygen saturation of $95 \%$ on room air. Physical examination revealed blepharoptosis and akinesia, however, no abnormalities were found in pulmonary and abdominal examinations. Electrocardiogram (ECG) suggested the possibility of myocardial infarction (Figure 1), while blood tests indicated disturbances in the serum myocardial enzyme spectrum. No obvious abnormalities were found in the coronary angiography (Figure 2). The patient did not consent to cardiovascular magnetic resonance (CMR) examination.

The patient was diagnosed with sintilimab-induced myocarditis based on the abnormal serum myocardial zymogram, abnormal ECG manifestations while presenting a normal coronary angiograph, and a correlation between the use of sintilimab and the onset of clinical symptoms. Sintilimab-induced myocarditis was considered a fourthdegree adverse event, and therefore the drug was permanently discontinued and methylprednisolone was administered on admission, meanwhile, it is supplemented with low-molecular heparin anticoagulation, aspirin to inhibit platelet aggregation, rosuvastatin calcium tablets to regulate blood lipid, isosorbide mononitrate to dilate blood vessels and other treatments. Due to the gradual improvement in the symptoms of dysphagia as well 


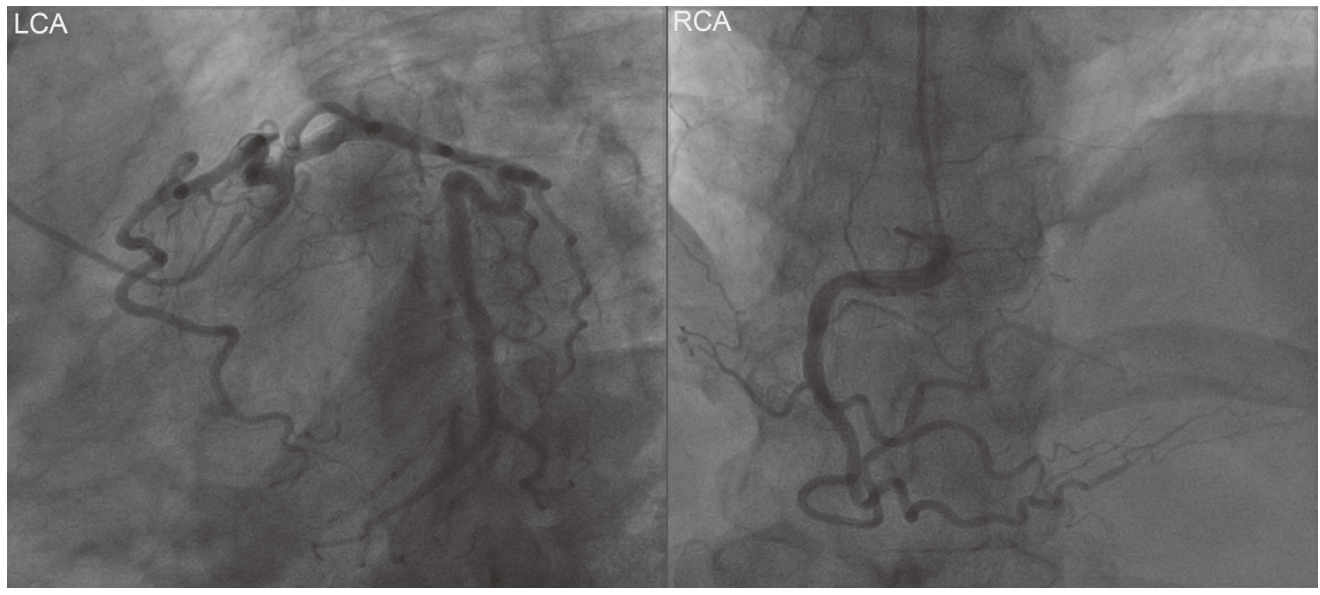

Figure 2 Coronary angiography did not show any vasculopathy. LCA, left coronary artery; RCA, right coronary artery.

as in the serum myocardial zymogram. Subsequently, the dose of methylprednisolone was gradually reduced from 80 to $40 \mathrm{mg} / \mathrm{d}$ (Figure 3). To reduce the side effects of methylprednisolone,Proton pump inhibitors protect the gastric mucosa,Vitamin D and calcium prevent osteoporosis, short-term use of zopicron or benzodiazepines may be required if patients have poor sleep quality.

The patient was discharged on day 13 after admission and the corticosteroid prednisolone was prescribed at $16 \mathrm{mg}$ daily for 15 days, followed by $8 \mathrm{mg}$ daily for 45 days. The patient showed good compliance during follow-up. However, subsequent chemotherapy again resulted in an increase in the patient's high-sensitivity troponin I (hs-TnI) levels (Figure 3A), and the patient returned to normal after methylprednisolone treatment. Apart from this, no other remarkable adverse events were reported throughout the treatment. At present, myocarditis has not re-occurred and lung cancer is at a stable stage.

All procedures performed in studies involving human participants were in accordance with the ethical standards of the institutional and/or national research committee(s) and with the Declaration of Helsinki (as revised in 2013). Written informed consent for publication was obtained from the patient.

\section{Discussion}

Immunotherapy is a promising and emerging line of therapy for malignancies, and in 2013, it was hailed by Science magazine as "breakthrough of the year" (27). Sintilimab is a humanized monoclonal IgG4 antibody that acts as an ICI against PD-1. It has been reported to prolong the survival of NSCLC patients. Unfortunately, immunoglobulinbased drugs are known to cause a series of irAEs which are different from traditional chemotherapy drugs. Currently, sintilimab is undergoing phase I, II, and III clinic trial in NSCLC patients $(26,28)$. To the best of our knowledge, this is the first report on immune myocarditis caused by sintilimab and the first review on ICI-induced myocarditis in lung cancer patients. The recent addition of sintilimab to the repertoire of anti-cancer agents along with the scarcity of its usage means there is a dearth of literature on its potential side-effects. This leaves the practitioners with very little reference when determining the optimal course of treatment.

The present study reviewed the case reports available in the literature for lung cancer patients who suffered from ICI-induced myocarditis. For the 20 cases reviewed, the clinical features, pathological types, and treatment of these patients were compiled (Table 1).

In the case study of our patient, ICI-induced myocarditis occurred on day 6 after receiving the third cycle of sintilimab, which is consistent with the timeframe of myocarditis reported in most cases (29). This highlights the observation that ICI-induced myocarditis is more likely to occur after using this drug for 2-3 cycles. Long-term application of this treatment did not appear to cease or lower the incidence of myocarditis as the patient showed symptoms even on day 4 of the $29^{\text {th }}$ cycle. Thus, clinicians should be wary of the potential side-effect in both shortand long-term usage of sintilimab. 
A

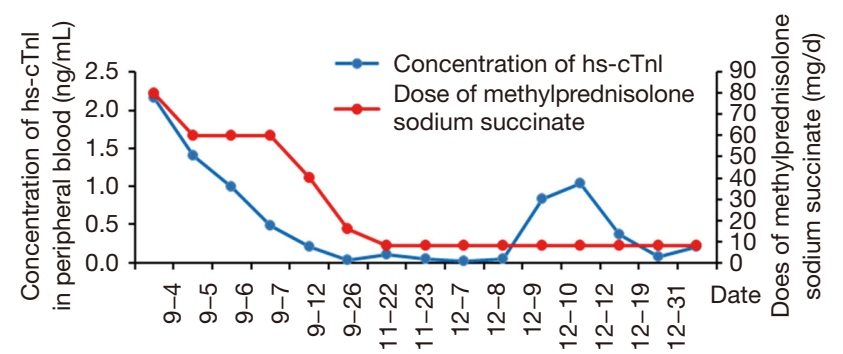

$\mathrm{C}$

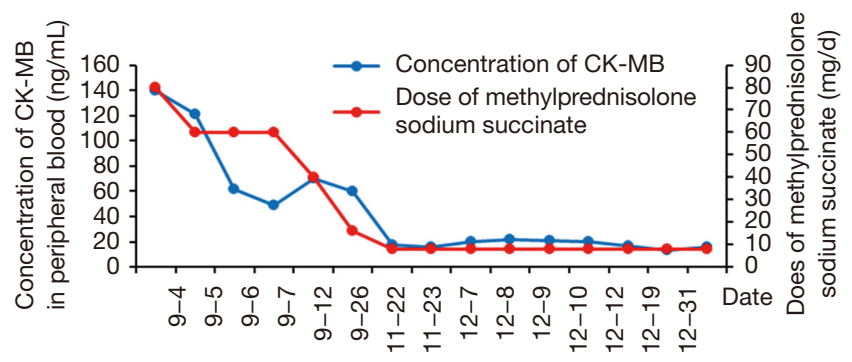

B

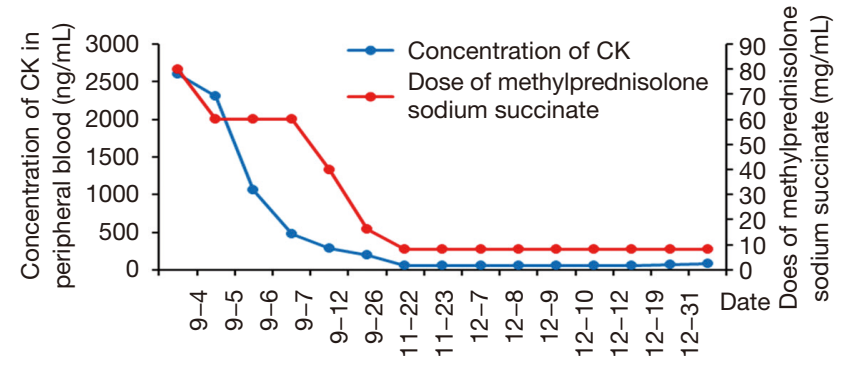

$\mathrm{D}$

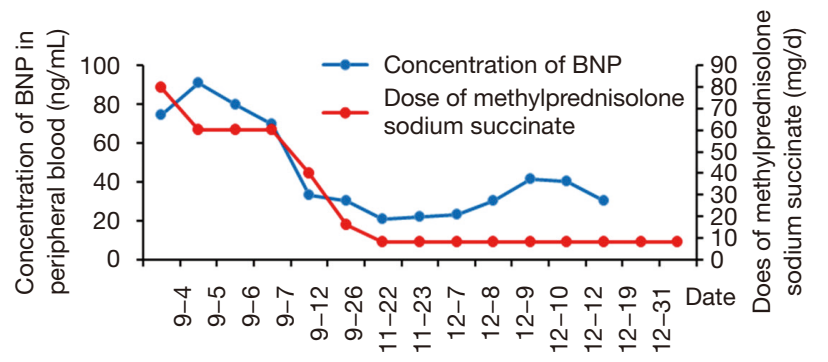

Figure 3 Changes in the serum myocardial markers during corticosteroid administration. (A) The time-dependent relationship between plasma hs-cTnI levels and corticosteroid dose. (B) The time-dependent relationship between serum CK levels and corticosteroid dose. (C) The time-dependent relationship between serum CK-MB levels and corticosteroid dose. (D) The time-dependent relationship between plasm BNP levels and corticosteroid dose. hs-cTnI, high sensitivity cardiac troponin I; CK, creatine kinase; CK-MB, creatine kinase MB; BNP, B-type natriuretic peptide.

Clinical manifestations of ICI-induced myocarditis may include typical symptoms (30), such as chest tightness, shortness of breath, chest pain, and exertional dyspnea. However, the patient examined in this case report did not present with these classical signs. Instead, he was admitted to the hospital complaining of dysphagia, hinting at the possibility of myasthenia gravis (14). Therefore, it is advisable to assess the serum acetylcholine receptor antibody levels upon admission to aid early diagnosis. Furthermore, accurate diagnosis of myocarditis is further complicated by the fact that some patients may not show any symptoms and present with a normal ECG pattern $(19,23)$. However, third degree atrioventricular block (AVB) may be a risk factor for increased mortality $(17,23)$. Early recognition of the signs of myocarditis exacerbation is the key to reducing mortality.

Since the ECG of a patient with myocarditis may have similar presentation to other acute cardiac syndromes such as myocardial infarction, an array of tests is required to make a definitive diagnosis. A coronary angiography may be considered. However, in our review of the literature, a statistical analysis of the coronary angiography in 10 patients found the results to be within normal limits, which was consistent with previous observations made by O'Neill et al. in 1985 (31). Therefore, we suggest that echocardiographic findings may complement the diagnosis of myocarditis if it is clinically correlated with extremely reduced left ventricular ejection fraction (LVEF), impaired diastolic function, cardiac hypertrophy, or myocarditis spots.

Among the various cardiovascular imaging modalities available, cardiac magnetic resonance (CMR) imaging is the first choice for the non-invasive diagnosis of myocarditis (32). The Lake Louise Criteria for CMR diagnosis of myocarditis includes three parameters: myocardial edema, hyperemia, and necrosis, these criteria correspond to abnormal findings in signal-enhanced T2weighted imaging, early gadolinium-enhanced (EGE) imaging, as well as on late gadolinium-enhanced (LGE) T1-weighted imaging. The presence of any two of the three diagnostic criteria may still support myocarditis with a diagnostic accuracy of $78 \%$, and sensitivity and specificity of $67 \%$ and $91 \%$, respectively, when clinically correlated (33). Recent meta-analyses showed that if only one of the criteria 
is met, the specificity is reduced to $78 \%$, but the diagnostic accuracy is improved to $83 \%$, while the sensitivity is increased to $78-80 \%$. Meanwhile, invasive examinations such as coronary angiography and endomyocardial biopsy may be avoided $(34,35)$. Therefore, it is recommended that patients should be assessed with CMR examinations.

Endomyocardial biopsy is the "gold standard" for the diagnosis of myocarditis. However, it is rarely performed due to its invasive nature and risk of complications such as major perforation (36). Among the 20 cases reviewed, only four patients received an endomyocardial biopsy test, of which one was a diagnostic autopsy $(9,14,20,23)$. The pathological sections showed scattered inflammatory lesions, necrosis of cardiomyocytes and granulated tissue, and infiltrating $\mathrm{T}$ cells and macrophages. Persistent inflammatory stimulation of the endocardium and the inferior wall myocardium is a characteristic manifestation of myocarditis (37), while giant cell and lymphocyte infiltration are the histologic markers of necrosis (38). Samantha et al. divided checkpoint inhibitor myocarditis into high grade ( $\geq 50 \mathrm{CD} 3+$ cells per high power field) and low grade $(\leq 50 \mathrm{CD} 3+$ cells per high power field $)$. The study showed that all patients with high grade myocarditis died (39) and statistical analyses demonstrated a strong correlation between endomyocardial biopsy pathology and death, suggesting that higher grade is associated with higher mortality. Recent reports indicate that the relatively distinctive histologic feature of ICI-induced myocarditis is diffuse lymphocytic infiltration. This conclusion needs to be further verified due to the low number of endomyocardial biopsy (40).

In most patients with ICI-induced myocarditis, standard response is to terminate immunosuppressive therapy and switch to high-dose corticosteroids (methylprednisolone or prednisone 1 to $2 \mathrm{mg} / \mathrm{kg} / \mathrm{d}$ ) to prevent further damage. If symptoms persist with no corresponding improvement in laboratory results, additional administration of immunosuppressants such as infliximab, rituximab, and mycophenolate mofetil should be considered (41). Abatacept, a cytotoxic T-lymphocyte-associated antigen 4 (CTLA-4) immunoglobulin, may also be considered (25). Corticosteroid therapy should be gradually reduced over a 4- to 6-week period. Statistical analyses of the literature revealed that the overall mortality of ICI-induced myocarditis in lung cancer patients was $23.8 \%(5 / 21)$, which was consistent with previous reports (42). The efficacy of corticosteroid therapy in the management of ICI-associated myocarditis was approximately $83.3 \%(15 / 18)$. It is believed that the use of methylprednisolone can help the patient through the acute phase of cardiotoxicity. As for whether hormone therapy affect the follow-up tumor treatment of sintilimab induced myocarditis, there are currently no relevant guidelines and reports, which require further clinical observations to obtain exact relevant conclusions.

Corticosteroid therapy is a double-edged sword which may induce new allergic reactions while treating other immune reactions $(43,44)$. Previous case reports have shown high levels of myocardial biomarkers such as $\mathrm{N}$ terminal pro-hormone B-type natriuretic peptide (NT-proBNP), high sensitivity (hs)-troponin, and creatine kinase (CK) $(10,18,20)$. In contrast, our lung cancer patient did not present with drastically altered levels of these biomarkers. This may be due to the early stage of his condition. Interestingly, during the course of treatment, the levels of hs-TnI, CK, and creatine kinase myocardial band (CK-MB) decreased steadily with the use of corticosteroids (Figure 3). A previous study reported that corticosteroids resulted in decreased levels of CK-MB compared to other biomarkers such as cardiac troponin I (cTnI), and this study showed similar changes (19). Therefore, patients may require specific dose adjustments of corticosteroids depending on the severity of the condition, and this will need to be verified by prospective experimental studies. In the absence of experience, Brahmer JR et al.'s report can be referred to achieve the purpose of individualized treatment (41).

In conclusion, this case reported the use of methylprednisolone to successfully treat a lung cancer patient suffering from ICI-induced myocarditis caused by sintilimab. Due to the life-threatening nature of this adverse event, particular attention is required when diagnosing lung cancer patients who present with myocarditis and are on a course of PD-1 inhibitors. Initial patient evaluation of potential cardiovascular toxicity should include ECG, troponin, BNP, chest CT, and so on. Baseline information may be useful when patients present with acute nonspecific symptoms and ambiguous diagnostic testing. This is particularly important as the clinical symptoms overlap with other cardiac syndromes. This, together with the novelty of the disease, and the absence of sufficient diagnostic evidence, makes ICI-induced myocarditis a difficult condition to diagnose and treat. However, due to the severity associated with this condition, it is imperative to continue research in this field to gain a better understanding, so as to improve diagnosis and treatment for these patients. Finally, increased 
awareness and close cooperation between respiratory specialists, oncologists, cardiologists, and ICU specialists are essential for the well-being of the patient.

\section{Patient perspective}

I trusts the doctor's choice of treatment at all times, even in the absence of cardiac biopsy pathology, has been proven to increase my probability of saving my life.

\section{Acknowledgments}

The authors acknowledge that the results of the ECG and coronary angiography were provided by the Huangdao District Hospital of Traditional Chinese Medicine.

Funding: This research project was subsidized by the Wu Jieping Medical Foundation (Approval number: 320.6750.19094-29).

\section{Footnote}

Reporting Checklist: The authors have completed the CARE reporting checklist. Available at http://dx.doi.org/10.21037/ apm-20-2449

Conflicts of Interest: All authors have completed the ICMJE uniform disclosure form (available at http://dx.doi. org/10.21037/apm-20-2449). The authors have no conflicts of interest to declare.

Ethical Statement: The authors are accountable for all aspects of the work and in ensuring that questions related to the accuracy or integrity of any part of the work are appropriately investigated and resolved. All procedures performed in studies involving human participants were in accordance with the the ethical standards of the institutional and/or national research committee(s) and Declaration of Helsinki (as revised in 2013). Written informed consent for publication was obtained from the patient.

Open Access Statement: This is an Open Access article distributed in accordance with the Creative Commons Attribution-NonCommercial-NoDerivs 4.0 International License (CC BY-NC-ND 4.0), which permits the noncommercial replication and distribution of the article with the strict proviso that no changes or edits are made and the original work is properly cited (including links to both the formal publication through the relevant DOI and the license). See: https://creativecommons.org/licenses/by-nc-nd/4.0/.

\section{References}

1. Caforio AL, Pankuweit S, Arbustini E, et al. Current state of knowledge on aetiology, diagnosis, management, and therapy of myocarditis: a position statement of the European Society of Cardiology Working Group on Myocardial and Pericardial Diseases. Eur Heart J 2013;34:2636-48, 2648a-2648d.

2. Haslam A, Prasad V. Estimation of the Percentage of US Patients With Cancer Who Are Eligible for and Respond to Checkpoint Inhibitor Immunotherapy Drugs. JAMA Netw Open 2019;2:e192535.

3. Wang DY, Salem JE, Cohen JV, et al. Fatal Toxic Effects Associated With Immune Checkpoint Inhibitors: A Systematic Review and Meta-analysis. JAMA Oncol 2018;4:1721-8.

4. Friedman CF, Proverbs-Singh TA, Postow MA. Treatment of the Immune-Related Adverse Effects of Immune Checkpoint Inhibitors: A Review. JAMA Oncol 2016;2:1346-53.

5. Johnson DB, Balko JM, Compton ML, et al. Fulminant Myocarditis with Combination Immune Checkpoint Blockade. N Engl J Med 2016;375:1749-55.

6. Totzeck M, Schuler M, Stuschke M, et al. Cardio-oncology - strategies for management of cancer-therapy related cardiovascular disease. Int J Cardiol 2019;280:163-75.

7. Raikhelkar J, Uriel N. Immune checkpoint inhibitor myocarditis. Curr Opin Cardiol 2019;34:303-6.

8. Inayat F, Masab M, Gupta S, et al. New drugs and new toxicities: pembrolizumab-induced myocarditis. BMJ Case Rep 2018;2018.

9. Matsuo K, Ishiguro T, Najama T, et al. Nivolumabinduced Myocarditis Successfully Treated with Corticosteroid Therapy: A Case Report and Review of the Literature. Intern Med 2019;58:2367-72.

10. Tan JL, Mugwagwa AN, Cieslik L, et al. Nivolumabinduced myocarditis complicated by complete atrioventricular block in a patient with metastatic nonsmall cell lung cancer. BMJ Case Rep 2019;12:e229963.

11. Gibson R, Delaune J, Szady A, et al. Suspected autoimmune myocarditis and cardiac conduction abnormalities with nivolumab therapy for non-small cell lung cancer. BMJ Case Rep 2016;2016:bcr2016216228.

12. Katsume $Y$, Isawa T, Toi $Y$, et al. Complete Atrioventricular 
Block Associated with Pembrolizumab-induced Acute Myocarditis: The Need for Close Cardiac Monitoring. Intern Med 2018;57:3157-62.

13. Matson DR, Accola MA, Rehrauer WM, et al. Fatal Myocarditis Following Treatment with the PD-1 Inhibitor Nivolumab. J Forensic Sci 2018;63:954-7.

14. Fukasawa Y, Sasaki K, Natsume M, et al. NivolumabInduced Myocarditis Concomitant with Myasthenia Gravis. Case Rep Oncol 2017;10:809-12.

15. Semper H, Muehlberg F, Schulz-Menger J, et al. Druginduced myocarditis after nivolumab treatment in a patient with PDL1- negative squamous cell carcinoma of the lung. Lung Cancer 2016;99:117-9.

16. Nierstedt RT, Yeahia R, Barnett KM. Unanticipated Myocarditis in a Surgical Patient Treated With Pembrolizumab: A Case Report. A A Pract 2020;14:e01177.

17. Khan A, Riaz S, Carhart R, Jr. Pembrolizumab-Induced Mobitz Type 2 Second-Degree Atrioventricular Block. Case Rep Cardiol 2020;2020:8428210.

18. Frigeri M, Meyer P, Banfi C, et al. Immune Checkpoint Inhibitor-Associated Myocarditis: A New Challenge for Cardiologists. Can J Cardiol 2018;34:92.e1-92.e3.

19. Tu L, Liu J, Li Z, et al. Early detection and management of immune-related myocarditis: Experience from a case with advanced squamous cell lung carcinoma. Eur J Cancer 2020;131:5-8.

20. Imai R, Ono M, Nishimura N, et al. Fulminant Myocarditis Caused by an Immune Checkpoint Inhibitor: A Case Report With Pathologic Findings. J Thorac Oncol 2019;14:e36-8.

21. Valenti-Azcarate R, Esparragosa Vazquez I, Toledano Illan $\mathrm{C}$, et al. Nivolumab and Ipilimumab-induced myositis and myocarditis mimicking a myasthenia gravis presentation. Neuromuscul Disord 2020;30:67-9.

22. Chauhan A, Burkeen G, Houranieh J, et al. Immune checkpoint-associated cardiotoxicity: case report with systematic review of literature. Ann Oncol 2017;28:2034-8.

23. Prevel R, Colin G, Cales V, et al. Third degree atrioventricular blockade during a myocarditis occurring under anti-PD1 : Case report and literature review. Rev Med Interne 2020;41:284-8.

24. Sakai T, Sasada S, Jyo C, et al. Acute myocarditis and pericarditis after nivolumab treatment in patients with non-small cell lung cancer. Ann Oncol 2017;28:IX90.

25. Salem JE, Allenbach Y, Vozy A, et al. Abatacept for Severe Immune Checkpoint Inhibitor-Associated Myocarditis. N Engl J Med 2019;380:2377-9.
26. Hoy SM. Sintilimab: First Global Approval. Drugs 2019;79:341-6.

27. Couzin-Frankel J. Breakthrough of the year 2013. Cancer immunotherapy. Science 2013;342:1432-3.

28. Yang Y, Wang Z, Fang J, et al. Efficacy and Safety of Sintilimab Plus Pemetrexed and Platinum as FirstLine Treatment for Locally Advanced or Metastatic Nonsquamous NSCLC: a Randomized, Double-Blind, Phase 3 Study (Oncology pRogram by InnovENT antiPD-1-11). J Thorac Oncol 2020;15:1636-46.

29. Escudier M, Cautela J, Malissen N, et al. Clinical Features, Management, and Outcomes of Immune Checkpoint Inhibitor-Related Cardiotoxicity. Circulation 2017;136:2085-7.

30. Läubli H, Balmelli C, Bossard M, et al. Acute heart failure due to autoimmune myocarditis under pembrolizumab treatment for metastatic melanoma. J Immunother Cancer 2015;3:11.

31. O'Neill D, McArthur JD, Kennedy JA, et al. Myocardial infarction and the normal arteriogram--possible role of viral myocarditis. Postgrad Med J 1985;61:485-8.

32. Jordan JH, D'Agostino RB, Jr., Hamilton CA, et al. Longitudinal assessment of concurrent changes in left ventricular ejection fraction and left ventricular myocardial tissue characteristics after administration of cardiotoxic chemotherapies using T1-weighted and T2-weighted cardiovascular magnetic resonance. Circ Cardiovasc Imaging 2014;7:872-9.

33. Friedrich MG, Sechtem U, Schulz-Menger J, et al. Cardiovascular magnetic resonance in myocarditis: A JACC White Paper. J Am Coll Cardiol 2009;53:1475-87.

34. Lagan J, Schmitt M, Miller CA. Clinical applications of multi-parametric CMR in myocarditis and systemic inflammatory diseases. Int J Cardiovasc Imaging 2018;34:35-54.

35. Kotanidis CP, Bazmpani MA, Haidich AB, et al. Diagnostic Accuracy of Cardiovascular Magnetic Resonance in Acute Myocarditis: A Systematic Review and Meta-Analysis. JACC Cardiovasc Imaging 2018;11:1583-90.

36. Guglin M, Nallamshetty L. Myocarditis: diagnosis and treatment. Curr Treat Options Cardiovasc Med 2012;14:637-51.

37. Aretz HT. Myocarditis: the Dallas criteria. Hum Pathol 1987;18:619-24.

38. Maisch B. Cardio-Immunology of Myocarditis: Focus on Immune Mechanisms and Treatment Options. Front Cardiovasc Med 2019;6:48.

39. Champion SN, Stone JR. Immune checkpoint inhibitor 
associated myocarditis occurs in both high-grade and lowgrade forms. Mod Pathol 2020;33:99-108.

40. Sobol I, Chen CL, Mahmood SS, et al. Histopathologic Characterization of Myocarditis Associated With Immune Checkpoint Inhibitor Therapy. Arch Pathol Lab Med 2020;144:1392-6.

41. Brahmer JR, Lacchetti C, Schneider BJ, et al. Management of Immune-Related Adverse Events in Patients Treated With Immune Checkpoint Inhibitor Therapy: American Society of Clinical Oncology Clinical Practice Guideline. J Clin Oncol 2018;36:1714-68.

42. Zhang L, Jones-O'Connor M, Awadalla M, et al.

Cite this article as: Bi H, Ren D, Wang Q, Ding X, Wang H. Immune checkpoint inhibitor-induced myocarditis in lung cancer patients: a case report of sintilimab-induced myocarditis and a review of the literature. Ann Palliat Med 2021;10(1):793802. doi: 10.21037/apm-20-2449
Cardiotoxicity of Immune Checkpoint Inhibitors. Curr Treat Options Cardiovasc Med 2019;21:32.

43. Kounis NG, Koniari I, Soufras GD, et al. Anaphylactic shock with methylprednisolone, Kounis syndrome and hypersensitivity to corticosteroids: a clinical paradox. Ital J Pediatr 2018;44:143.

44. Real M, Barnhill MS, Higley C, et al. Drug-Induced Liver Injury: Highlights of the Recent Literature. Drug Saf 2019;42:365-87.

(English Language Editor: J. Teoh) 